\title{
Simulations and experiments on magneto-optical diffraction by an array of epitaxial $\mathrm{Fe}(001)$ microsquares
}

\author{
P. García-Mochales, ${ }^{\text {a) }}$ J. L. Costa-Krämer, G. Armelles, and F. Briones \\ Instituto de Microelectrónica de Madrid, CNM, CSIC. Isaac Newton 8 (PTM), Tres Cantos 28760 Madrid, \\ Spain \\ D. Jaque, J. I. Martín, ${ }^{\text {b) }}$ and J. L. Vicent \\ Depto de Física de Materiales, C.C. Físicas, Universidad Complutense, 28040 Madrid, Spain
}

(Received 20 May 2002; accepted 19 August 2002)

\begin{abstract}
Magneto-optic (MO) experimental and computational studies are reported on arrays of epitaxial $\mathrm{Fe}(001)$ microsquares. Measuring the transverse Kerr signal, in both reflected and diffracted spots, when the array is exposed to a rotating magnetic field allows the quantification of the magnetization inhomogeneties within the microsquares. The MO signal at diffracted spots is extremely sensitive to magnetization inhomogeneities, while working at a large constant field amplitude eliminates uncertainties due to domain-wall movements. The presence of anomalous peaks at the diffracted MO response is unambiguously assigned to the matching of inhomogeneities of the magnetization distribution to the corresponding pattern periodicity. Interaction between microsquares appears as a second-order effect. (C) 2002 American Institute of Physics. [DOI: 10.1063/1.1514388]
\end{abstract}

The improvement in methods for epitaxial growth and lithographic techniques allows the control of the shape and size of magnetic elements in the nanometer range and offers the possibility to place them in regular arrays. ${ }^{1}$ These arrays of magnetic micro/nano-objects permit the study of technologically relevant properties such as the magnetic interaction between them or "domino" magnetization switching of the array elements. Magneto-optical (MO) techniques are very suitable to study such elements due to their sensitivity and to the possibility of using light reflected and diffracted by the periodic array to analyze the magnetic behavior of the system. $^{2-9}$ As opposed to reflected light, a system modeling is required to fully understand the information carried by diffracted beams. In this letter, we present a MO study of single-crystalline $\mathrm{Fe}(001)$ arrays of square microelements or microtiles. A transverse Kerr configuration is used and a rotating magnetic field $\mathbf{H}$ is applied in the plane of the sample, MO torque (MOT) setup. ${ }^{9}$ Comparing experiments with simulations has allowed the identification of specific features on diffracted beams that are due to magnetization inhomogeneities within the microtiles.

The samples used in the presented study are $30 \mathrm{~nm}$ thick single-crystal $\mathrm{Fe}(001)$ film grown on a $100 \mathrm{~nm} \mathrm{MgO}$ buffer layer on $\operatorname{GaAs}(001)$ substrates. The samples are patterned into arrays of microtiles with different periods $T(=2.5,5,10$ $\mu \mathrm{m})$ and separations $w(=0.2,0.6 \mu \mathrm{m})$ by a combined use of electron-beam lithography and ion-beam etching. ${ }^{1,9}$ In this study, epitaxial Fe hard axes [110] are parallel to the square edge. The whole field patterned is a $250 \times 250 \mu \mathrm{m}^{2}$ square and is illuminated with a laser spot of about $140 \mu \mathrm{m}$ diameter. Depending on the pattern element sizes, from hundreds to thousands of individual microtiles are illuminated, obtaining a diffraction pattern. The laser was $p$ polarized and the incidence plane is parallel to one of the square edges ( $x$ axis).

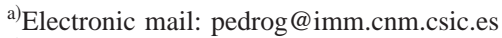

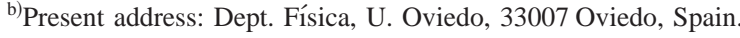

In Fig. 1, the MOT signal (i.e., the variation of the $p$-polarized reflected light intensity as a function of the angle between $\mathbf{H}$ and the $y$ axis) for two typical samples [ $T$ $=5 \mu \mathrm{m}$ and $w=0.2$ and $0.6 \mu \mathrm{m}$ for Figs. 1(a) and 1(b), respectively] taken at different diffraction orders is presented. The intensity of the rotating magnetic field can be continuously adjusted in the $0-0.3 \mathrm{~T}$ range. The zero-order signals are very similar in both samples, but the diffraction signals are very different. In particular, the first- and secondorder diffraction signals show secondary peaks when $\mathbf{H}$ is parallel to the $x$ axis, but they decrease as the separation between tiles increases. This could be attributed to a signature of magnetic interaction between neighboring tiles. In addition, the relative peak height decreases as the field magnitude (and, consequently, the magnetic homogeneity of the sample) increases. They also decrease slightly when the tile size increases.

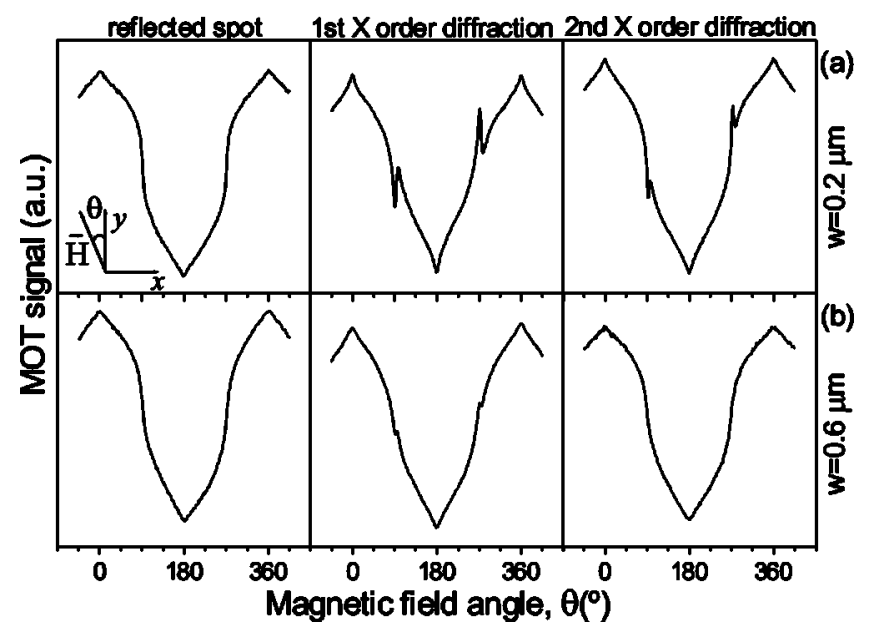

FIG. 1. Experimental MOT results: Kerr intensity $I_{\text {Kerr }}^{(n, 0)}$ for diffraction order $n=0,1$, and 2 as function of the magnetic field angle. Samples shown have a period $T=5 \mu \mathrm{m}$ and a field applied $H=0.085 \mathrm{~T}$. (a) Tile separation $w$ $=0.2 \mu \mathrm{m}$. (b) $w=0.6 \mu \mathrm{m}$. 
In order to understand the different behaviors of diffracted and reflected beams, it is necessary to have, on the one hand, a model which describes the magnetic behavior of the tiles and, on the other, a good description of the MO properties of the regular array. The use of a large rotating constant field eliminates domain-wall propagation inside tiles, simplifying the magnetization behavior analysis. It is assumed that all the array elements behave in the same way, and the reflection coefficient $r_{p p}$ only depends on the $y$-component magnetization $m_{y} .{ }^{10}$ In general, $r_{p p}$ is not constant within the tile due to the spatial distribution of $m_{y}(x, y)$. To calculate the diffracted intensities that depend on magnetization, the Fraunhofer diffraction theory is used for a two-dimensional grating of square motif, taking into account the spatial dependence of $r_{p p}$. Keeping only terms that depend on $m_{y}$ and neglecting terms higher than linear in $m_{y}$, the Kerr intensities at diffraction maxima read as

$$
I_{\text {Kerr }}^{(n, m)} \approx \operatorname{Re}\left(B \int_{-a / 2}^{a / 2} \int_{-a / 2}^{a / 2} m_{y}(x, y) e^{i 2 \pi(n x+m y) / T} d x d y\right),
$$

where $(n, m)$ are the $x$ and $y$ direction diffraction orders, $a$ is the length of the square tile $(T=a+w)$, and $B$ is a complex parameter that depends on the material, light wavelength, and incidence angle. To obtain magnetization distributions within the individual tiles at a given position of the rotating $\mathbf{H}$ field, micromagnetic calculations ${ }^{11}$ have been performed. Due to the size of tiles, two-dimensional simulations with three-dimensional spins have been carried out. The behavior of a single tile under a rotating field was calculated instead of an array of interacting tiles. With the $m_{y}(x, y)$ information, the different diffracted Kerr intensities $I_{\text {Kerr }}^{(n, m)}$ are calculated. The results of such calculation for the samples of Fig. 1 are presented in Figs. 2(a) and 2(b). While the reflected intensity $\left(\left\langle m_{y}\right\rangle\right)$ shows a good agreement between experimental results and simulation, this is not the case for diffracted intensities. They show the same features as the experimental results but several times larger. As in the experiments, secondary peaks decrease in height as the separation between tiles increases. As mentioned, herein, it was tempting at first to interpret the features observed in the diffracted spots and their behavior as a signature of magnetic interaction between neighboring tiles. However, these features and its evolution with the intertile distance is reproduced by the isolated tiles simulation and it should not then be attributed to a decrease of the intertile interaction. The origin of this decrease is mainly optic, associated with different diffraction geometries and not related with different magnetization distributions for different tile sizes or separations.

To find the physical origin of these secondary peaks the magnetization distributions obtained from the simulations are analyzed in detail. In Figs. 3(a)-3(d), several magnetization distributions within the tile for selected field orientations have been depicted (for the case of $a=4.8 \mu \mathrm{m}$ ). While the magnetization inside the tile follows the $\mathbf{H}$ field direction, along the tile edge the magnetization remains parallel to the border. When the $\mathbf{H}$ field crosses the hard $x$ axis, the magnetization close to the edges along the $y$ axis switches: Most of the tile magnetization is parallel to the $x$ axis, i.e., $m_{y}(x, y)$ $\sim 0$, except by the $y$ edges. In this situation, the lateral inhoDownloaded 15 Feb 2010 to 161.111 .180 .191 . Redistribution subje

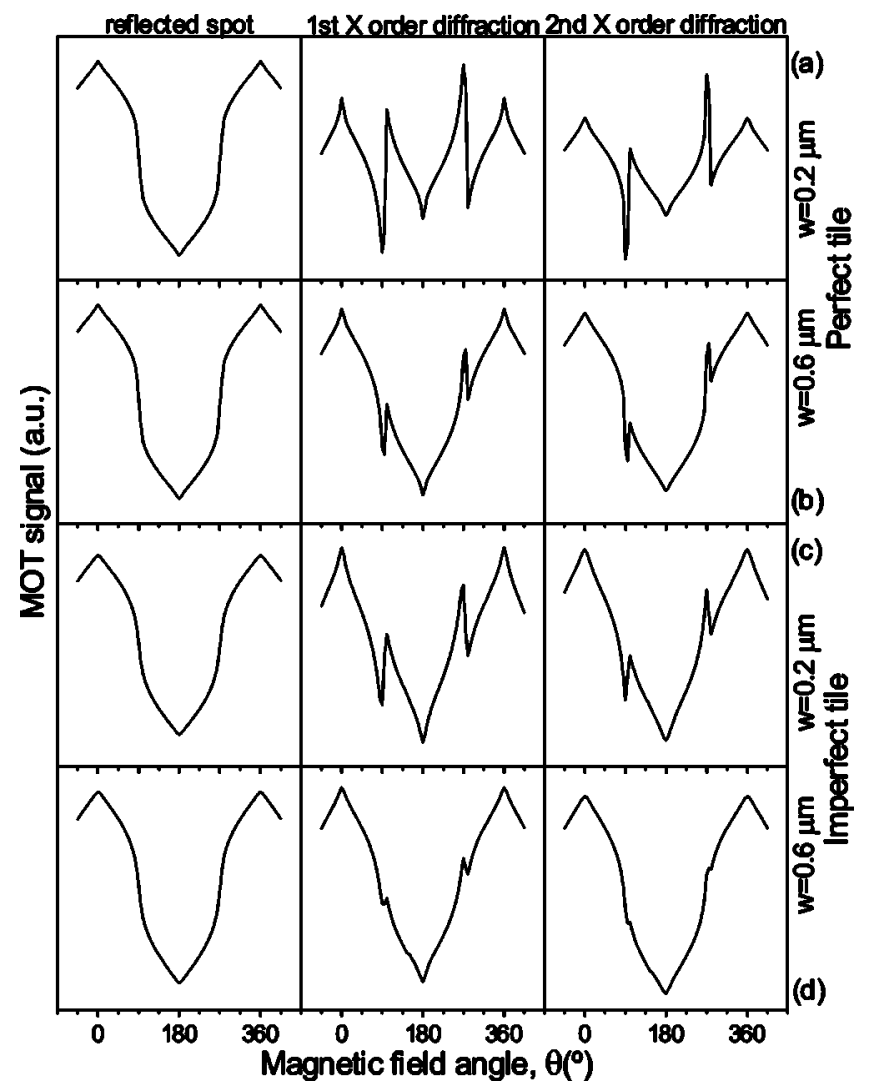

FIG. 2. Simulated MOT results for the same cases shown in Fig. 1. (a) and (b) Results corresponding to perfect square tiles $(w=0.2$ and $0.6 \mu \mathrm{m}$, respectively). (c) and (d) Results from imperfect square tiles simulations ( $w$ $=0.2$ and $0.6 \mu \mathrm{m})$.

mogeneities of the magnetization dominates the MOT signal [obtained from Eq. (1)]. Their abrupt change of direction with the rotating field produces the abrupt change observed on the spot intensities. When a larger $H$ field is applied, the homogeneity of the magnetization increases, decreasing the border effect and the height of the secondary peak. Therefore, these features should be very sensitive to the distribution of the magnetization along the edges, i.e., to the presence of defects. Microfabricated tiles do not have flat edges due to the ion milling process plus mask inhomogeneities. Additionally, some oxidation at the edges, presence of impurities, etc., can not be completely ruled out. These possible "defects" have been introduced into the simulation through the tile shape: Imperfect tiles with roughness, round corners, and irregular edges. The simulation of the MOT curves performed with these approximations is presented in Figs. 2(c) and 2(d), showing that these imperfect tiles have no noticeable effect on the reflected spot signal (on the $\left\langle m_{y}\right\rangle$ ). On the contrary, the anomalous peak at the MOT signal produced when the field crosses the $x$ axis [see Figs. 3(e)-3(h)] is strongly reduced due to local closure domains induced by roughness that facilitates the rotation of the magnetization near the borders.

Due to the strength of the field used, signs of the interaction between tiles found in other magneto-optical Kerr effect studies on tiles ${ }^{12}$ are concealed. To check how the interaction between tiles might influence the main features, a simulation of a $3 \times 3$ array of perfect tiles $(T=5 \mu \mathrm{m}$; $w=0.2 \mu \mathrm{m})$ has been performed. The MOT signals of this to AIP license or copyright; see http://apl.aip.org/apl/copyright.jsp 


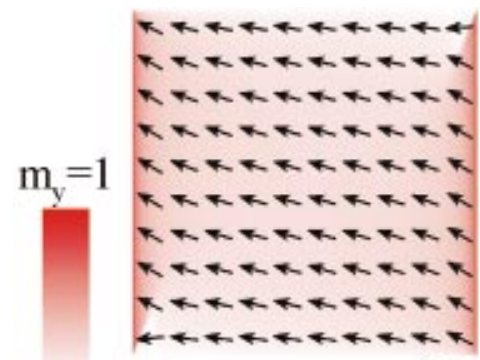

(a)

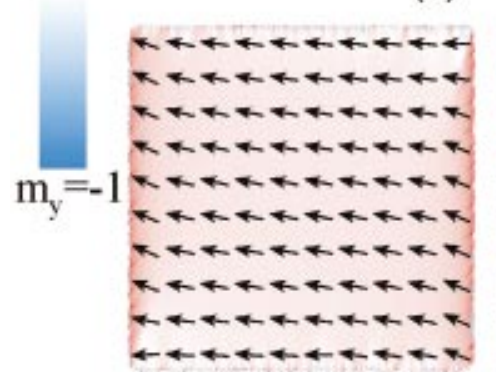

$\overrightarrow{\mathrm{H}}$

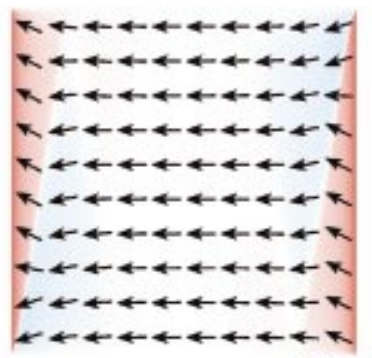

(b)

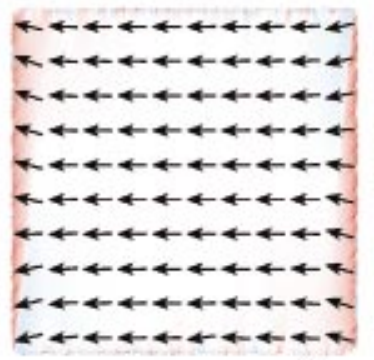

(f)

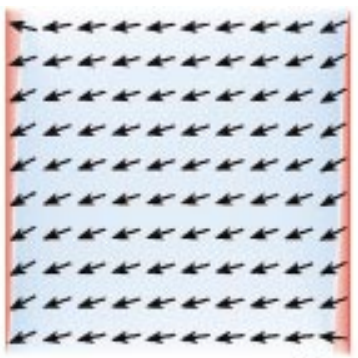

(c)

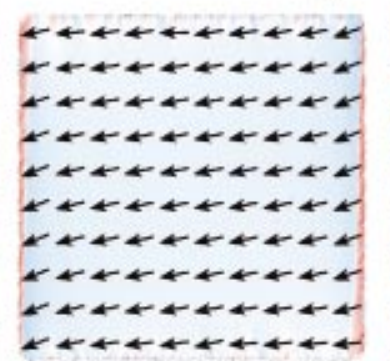

(g)

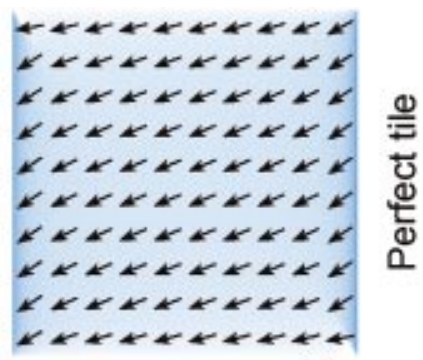

(d)

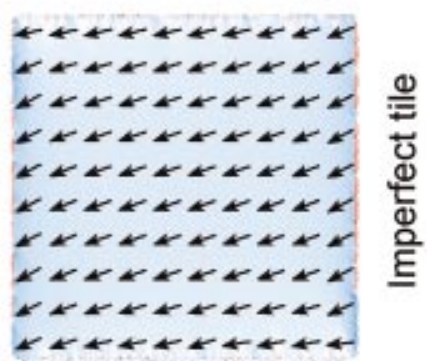

(h)

FIG. 3. (Color) $\mathrm{Fe}(001)$ tile magnetization distributions for selected field directions ( $\theta=85^{\circ}, 90^{\circ}, 95^{\circ}$, and $100^{\circ}$, respectively) obtained from micromagnetic simulations: $m(x, y)$ is represented with arrows; $m_{y}(x, y)$ with the color scale. Cases depicted correspond to those shown in Fig. 2: (a)-(d) for the perfect square tile simulation; (e)-(h) for the imperfect tile simulation. Note the sudden switch of the magnetization at the $y$ edges between (c) and (d) for the perfect tile and $(\mathrm{g})$ and $(\mathrm{h})$ for the imperfect tile.

simulation are similar to those shown in Figs. 2(a) and 2(b), but secondary peaks on diffraction spots are slightly lower (around 10\%). Interactions increase the homogeneity of the magnetization, helping a softer change of the border magnetization, but they are a second-order effect. However, in the light of the $3 \times 3$ perfect array result, differences between simulations of imperfect single tiles and experiments are probably due to the interaction effect.

In summary, MOT experiments and simulations for $\mathrm{Fe}(001)$ tile arrays are reported. While the reflected spot carries information about the average magnetization within the tiles just as in conventional Kerr magnetometry, the diffracted spots carry information about the different magnetization distribution moments (Fourier transformed) within the tiles. As such, they are more sensitive to inhomogeneities of the magnetization distribution than the reflected spot. Interaction between tiles manifests as a second-order effect. Changes on the diffracted spots signals of samples with different tile separation are mainly due to the different optical characteristic and not to changes on the magnetization distribution due to the intertile interaction.

The authors are grateful to A. Cebollada and C. Martínez-Boubeta for growing the thin film. Work supported by the Spanish CICYT under Contract Nos. MAT99-0724 and MAT99-1063. One of the authors (P.G.-M.) is supported by the Comunidad de Madrid through its postdoctoral program.

${ }^{1}$ J. I. Martín, Y. Jaccard, A. Hoffman, J. Nogués, J. M. George, J. L. Vicent, and I. K. Schuller, J. Appl. Phys. 84, 411 (1998).

${ }^{2}$ O. Geoffroy, D. Givord, Y. Otani, B. Pannetier, A. D. Santos, M. Schlenker, and Y. Souche, J. Magn. Magn. Mater. 121, 516 (1993).

${ }^{3}$ Y. Souche, M. Schlenker, and A. D. Dos Santos, J. Magn. Magn. Mater. 140, 2179 (1995).

${ }^{4}$ M. Hanson, C. Johansson, B. Nilsson, P. Isberg, and R. Wäppling, J. Appl. Phys. 85, 2793 (1999).

${ }^{5}$ P. Vavassori, V. Metlushko, M. Grimsditch, B. Ilic, P. Neuzil, and R. Kumar, Phys. Rev. B 61, 5895 (2000).

${ }^{6}$ T. Schmitte, T. Schemberg, K. Westerholt, and H. Zabel, J. Appl. Phys. 87, 5630 (2000).

${ }^{7}$ I. Guedes, N. J. Zaluzec, M. Grimsditch, V. Metlushko, P. Vavassori, B. Ilic, P. Neuzil, and R. Kumar, Phys. Rev. B 62, 11719 (2000).

${ }^{8}$ D. Jaque, J. I. Martín, G. Armelles, J. L. Costa-Krämer, F. Briones, and J. L. Vicent, J. Appl. Phys. 91, 382 (2002).

${ }^{9}$ G. Armelles, J. L. Costa-Krämer, J. I. Martin, J. V. Anguita, and J. L. Vicent, Appl. Phys. Lett. 77, 2039 (2000).

${ }^{10}$ M. E. Brubaker, E. R. Moog, C. H. Sowers, J. Zak, and S. D. Bader, J. Magn. Magn. Mater. 103, L7 (1992).

${ }^{11}$ OOMMF public code $1.1 \mathrm{~b}$; http://math.nist.gov/oommf

${ }^{12}$ J. L. Costa-Krämer, J. I. Martín, J. L. Menéndez, A. Cebollada, J. V. Anguita, F. Briones, and J. L. Vicent, Appl. Phys. Lett. 76, 3091 (2000). 